\title{
Female sacrococcygeal pilonidal sinus features and EQ-5D life quality survey and body image survey results: a clinical study
}

\author{
๑DMehmet Saydam ${ }^{1}$, \Mutlu Şahin ${ }^{2}$ \\ ${ }^{1}$ Department of General Surgery, Institute of Health Sciences, Dışkapı Training and Research Hospital, Ankara, Turkey \\ ${ }^{2}$ Department of General Surgery, Institute of Health Sciences, Keçiören Training and Research Hospital, Ankara, Turkey
}

Cite this article as: Saydam M, Şahin M. Female sacrococcygeal pilonidal sinus features and EQ-5D life quality survey and body image survey results: a clinical study. Anatolian Curr Med J 2021; 3(1); 31-35.

\begin{abstract}
Aim: Pilonidal sinus disease (PSD) is not common in female patients. It is seen in adult women 2.2 to 4 times less than men. The most common age range is between 10 and 40. To date, there has been no randomized or retrospective study in the literature representing the general characteristics of women-specific pilonidal sinus cases and the quality of life questionnaire (EQ-5D) and body image questionnaire (BIQ) results. The purpose of this study is to show the general features, treatment principles and body image and quality of life (EQ-5D) survey results of the female pilonidal sinus cases.

Material and Method: A retrospective clinical study was conducted with 29 women who were admitted to our clinic between June 2013 and May 2016 and treated with surgery by the pre-diagnosis of sacrococcygeal PSD. Patients received face-to-face body image questionnaire (BIQ) and quality of life questionnaire (EQ-5D) at the $2^{\text {nd }}$ postoperative week.

Results: The mean age was $27.07 \pm 5.63$ years. BIQ cosmetic and self-confidence scores differ significantly between at least two groups ( $\mathrm{p}=0.042$ and $\mathrm{p}=0.021$, respectively) in the favor of primer midline closure (PMC) group. As a result of bilateral comparisons made for the BIQ cosmetic score, there was a significant difference between the Limberg- $\mathrm{Z}$ plasty and the Karydakis group ( $\mathrm{p}=0.039)$.

Conclusion: Female patients who underwent PMC had better satisfaction with the appearance of their scar. The treatment of female PSD with minimally invasive techniques and primary closure techniques seems to be the best option for the selected patients.
\end{abstract}

Keywords: Pilonidal sinus disease, cosmesis, primer midline closure

\section{INTRODUCTION}

Pilonidal sinus disease (PSD) is a common and acquired disease especially of young male adults. It has an estimated incidence of $26 / 100,000$ in the general population (1). It is seen in adults women 2.2 to 4 times less than men (2). The most common age range is between 10 and 40 (3). Hair follicles mostly penetrate one or more sinus walls in the sacrococcygeal region, followed by an acute or subacute chronic infection, and this clinical condition is called PSD (4). PSD is generally a surgical disease and the main principles in surgical treatment are to minimize the amount of postoperative pain and wound care, recurrence rate, and to ensure that the patient returns to daily life as soon as possible. To date, many options for surgical treatment of the disease have been developed and described, but there is no consensus about treatment yet, since all surgical methods produce various recurrence rates. Formation of pilonidal sinus in women seems quite difficult due to the nature of the disease. In many studies, female gender is only between $1-5 \%$ of patients (1). To date, there has been no randomized or retrospective study in the literature representing the general characteristics of women-specific pilonidal sinus cases and the quality of life questionnaire (EQ-5D) and body image questionnaire (BIQ) results. The purpose of this study is to show the general features, treatment principles and body image and quality of life (EQ-5D) survey results of the female pilonidal sinus cases.

\section{MATERIAL AND METHOD}

A retrospective clinical study was conducted with patients who were admitted to the general surgery service of Ankara Mevki Military Hospital between June 
2013 and May 2016. A total of 29 women patients treated with surgery by the pre-diagnosis of sacrococcygeal PSD in this period were included in the study after obtaining the Ethics Committee Approval. Detailed files and contact numbers of all operated patients were obtained from the hospital archive. The inclusion criteria were accepted as all female patients who were operated between dates in question and agreed to participate in the study. The exclusion criterias were accepted as female patients who did not want to participate in the study and were treated with minimally invasive technique such as fenolisation and fibrin glue applications. In addition, when the patients were discharged, they were told that they would be interviewed face to face in the second postoperative week. All patients received face-to-face body image questionnaire (BIQ) and quality of life questionnaire (EQ-5D) at the $2^{\text {nd }}$ postoperative week. All patients had detailed information about the study and approved consent forms were signed to participate in the study. In addition, patients' age, body mass index (BMI), comorbidity, type of surgical procedure, and postoperative complications were also enrolled.

\section{Getting and Evaluating the Survey Results}

Questionnaires were used to compare the postoperative quality of life, body image and cosmesis of patients who underwent primary midline closure (PMC) and flap methods (Karyadakis, Modified Limberg). All the questionnaires were applied at the postoperative $2^{\text {nd }}$ week (early period) and the information was collected face to face. Quality of life was evaluated using the EuroQol-5 Dimension Questionnaire (EQ-5D ${ }^{\mathrm{mm}}$ ), which consists of six questions. The first five questions cover the descriptive system of the survey (mobility, self-care, routine activities, pain/discomfort and anxiety/depression). The purpose of the sixth question is to enable self-determination of the person's health. In this section, the person marks a visual analog scale to evaluate the patient's current health status, giving a value between 0 (worst) and 100 (maximum wellbeing). The secondary questionnaire of this study is about cosmesis and has been evaluated by BIQ $(5,6)$. The BIQ consists of questions that investigate patients' attitudes towards their physical appearance [body image scale (BIS), questions 1 to 5], degrees of satisfaction with scar appearance [cosmesis scale (CS) questions 6-8] and selfconfidence (questions 9-10). In this survey, a higher score indicates that patient satisfaction has increased.

\section{Statistical Analysis}

The suitability of the numerical variables obtained within the scope of the study to normal distribution was examined by Shapiro-Wilks test. In the summarization of the numerical variables, the mean \pm standard deviation and the median (minimum; maximum) descriptive statistics were used. The type of surgery, which is the categorical variable, is summarized by number and percentage.
Kruskal Wallis test was used to compare EQ-5D (index, daily health) and BIQ (image, cosmetics, self-confidence) scores by type of surgery. In double comparisons, Dunnbonferroni corrected test result was given. The relationship of EQ-5D and VIA scores with age, BMI and volume was examined by Spearman rho rank correlation coefficient. IBM SPSS Statistics for Windows, Version 21.0 (Armonk, NY: IBM Corp.) was used for statistical analysis. Statistical significance level was accepted as $\mathrm{p}<0.05$.

\section{RESULTS}

Descriptive information of the patients in the study is given in Table 1. The mean age was $27.07 \pm 5.63$ years, BMI average was $27.65 \pm 4.02 \mathrm{~kg} / \mathrm{m}^{2}$. Postoperative seroma was observed in 5 patients in total. One of them was in PMC group and 4 of them were in flap group. Postoperative wound dehiscence was observed in two patients, one of which was with flap operation and one was PMC operation. In total, 2 patients had superficial surgical site infection, one of which was with PMC and the other was with flap operation. Skin necrosis was not observed.

\begin{tabular}{|lcc|}
\hline \multicolumn{3}{|l|}{ Table 1. Demographics of the patients } \\
\hline & Mean \pm SD & Median (min; max) \\
\hline Age (year) & $27.07 \pm 5.63$ & $28(18 ; 39)$ \\
Volume $(\mathrm{x})$ & $16.52 \pm 10.80$ & $12(4 ; 50)$ \\
BMI $\left(\mathrm{kg} / \mathrm{m}^{2}\right)$ & $27.65 \pm 4.02$ & $28.2(19.5 ; 34.2)$ \\
Type of surgery & $\mathbf{n}(\%)$ \\
Primer midline closure & $10(34.5)$ \\
Karydakis & $14(48.3)$ \\
Limberg-Z plasty & $5(17.2)$ \\
Complication & $\mathrm{n}(\%)$ \\
Seroma & $5(17.24)$ \\
Wound dehiscence & $2(6.89)$ \\
Surgical site infection & $2(6.89)$ \\
\hline
\end{tabular}

Quality of life questionnaire (EQ-5D) index, answers to question 6 and BIQ image scores were statistically significantly similar according to the type of surgery ( $>0.05)$ (Table 2). BIQ cosmetic and self-confidence scores differ significantly between at least two groups ( $p=0.042$ and $p=0.021$, respectively). As a result of the bilateral comparisons made for the BIQ cosmetic score, there was a significant difference between the Limberg- $Z$ plasty and the Karydakis group $(\mathrm{p}=0.039)$, whereas other bilateral comparisons were not significant $(p>0.05)$. In binary comparisons made in surgery type groups for BIQ self-confidence score, the score obtained in the Limberg-Z plasty group was found to be significantly lower than that obtained in the Karyadakis group $(\mathrm{p}=0.018)$. There was no statistically significant difference between PMC and Karydakis and Limberg- $Z$ plasty surgery types in terms of $\mathrm{BIQ}$ self-confidence score $(\mathrm{p}>0.05)$. No statistically significant correlation was found between EQ-5D and BIQ scores and variables of age, BMI and volume ( $p>0.05)$ (Table 3). 


\begin{tabular}{|c|c|c|c|c|c|}
\hline \multicolumn{6}{|c|}{ Type of Surgery } \\
\hline & $\begin{array}{l}\text { Primer midline closure } \\
\text { median (min; max) }\end{array}$ & $\begin{array}{c}\text { Karydakis } \\
\text { median (min; max) }\end{array}$ & $\begin{array}{l}\text { Limberg-Z plasty } \\
\text { median (min; max) }\end{array}$ & $\mathrm{X}^{2}$ & $\mathbf{p}$ \\
\hline EQ-5D index & $0.828(0.828 ; 0.828)$ & $0.828(0.755 ; 1.000)$ & $0.828(0.755 ; 0.828)$ & 1.900 & 0.387 \\
\hline EQ-5D percent_Your Health Today? & $90(80 ; 95)$ & $90(75 ; 95)$ & $90(80 ; 95)$ & 0.940 & 0.625 \\
\hline VIA image & $17.5(15 ; 20)$ & $19.5(15 ; 20)$ & $17(15 ; 20)$ & 4.388 & 0.111 \\
\hline VIA cosmesis & $18(17 ; 22)^{\mathrm{a}, \mathrm{b}}$ & $20(16 ; 22)^{b}$ & $17(9 ; 20)^{\mathrm{a}}$ & 6.359 & 0.042 \\
\hline VIA self-confidence & $18(17 ; 18)^{a, b}$ & $18(16 ; 20)^{\mathrm{b}}$ & $17(13 ; 17)^{\mathrm{a}}$ & 7.750 & 0.021 \\
\hline
\end{tabular}

\begin{tabular}{|c|c|c|c|}
\hline & Age (year) rho; $p$ & Volume $\left(\mathrm{cm}^{3}\right)$ rho; $p$ & BMI $\left(\mathrm{kg} / \mathrm{m}^{2}\right)$ rho; $\mathrm{p}$ \\
\hline EQ-5D index & $-0.257 ; 0.179$ & $-0.273 ; 0.153$ & $-0.266 ; 0.162$ \\
\hline EQ-5D percent_Your Health Today? & $-0.046 ; 0.818$ & $0.026 ; 0.895$ & $0.241 ; 0.218$ \\
\hline VIA image & $0.282 ; 0.138$ & $0.293 ; 0123$ & $-0.015 ; 0.938$ \\
\hline VIA cosmesis & $-0.195 ; 0.312$ & $0.230 ; 0.231$ & $-0.230 ; 0.229$ \\
\hline VIA self-confidence & $0.319 ; 0.091$ & $-0.170 ; 0.379$ & $-0.258 ; 0.176$ \\
\hline
\end{tabular}

\section{DISCUSSION}

Pilonidal sinus disease (PSD) was described for the first time in 1833 by Herbert Mayo (7). The incidence of the PSD was 29/100.000 in the last two decades but nowadays $48 / 100.000$ (8). In a large number of case study by Duman et al. (9) conducted, they found $0.37 \%$ female ratio, and $6.23 \%$ male ratio who were candidate to be students or officials of the state's schools and institutions in Turkey. The PSD is more frequently seen in people of Middle East, and Mediterranean countries (10). Although male/ female ratio in some adult series is considerably high, this ratio tends to lower value such as $1 / 1.4$ in pediatric group (11).

A lot of risk factors such as excessive hair growth, poor hygiene, obesity, working conditions (sitting for long time) and family history were accused for possible etiopathogenesis of PSD. PSD is more common in darkskinned and brunette persons, and it has actually an important role in the etiology (12). All female patients who were involved to our study had dark hair and skin and excess body hair. Some authors determined the relationship between obesity and PSD (13). Our BMI mean was is $27.65 \pm 4.02 \mathrm{~kg} / \mathrm{m}^{2}$ and we could not find a significant difference between obesity and other criterias. However, as a clinical experience we observed that pilonidal sinus volume increased with obesity. It is thought that the reason for the statistically insignificant reason is that our number of cases is low and the criteria of selection is very narrow.

The primary endpoint in this study were quality-of-life measures, and cosmesis which are the important parameters for determining the postoperative satisfaction of females who may accept such disease more reluctantly comparing males. The current study did not establish any surgical technique superior to each other in terms of quality of life. However, patient satisfaction is a comprehensive topic with lots of components, such as patient's expects from the surgery, and the outcome that might have resulted if the other technique had been used for the patient, or anxiety of possible reccurant disease. Standardized methods of measuring patient satisfaction should be used in a randomized clinical trial with large sample size to compare satisfaction between female patients undergoing any surgical technique for PSD. On the other hand when we analyse the cosmesis via BIQ, cosmetic and self-confidence scores differ significantly between at PMC and other flap techniques. This statistic is thought to be due to the fact that the patients undergoing PMC have smaller PSD volume than those who underwent other flap operations, and consequently a more acceptable surgical scar which may be the most important thing for a female in such age group.

There is no consensus on the best treatment of PSD. A study conducted in Turkey showed that $83 \%$ of the surgeons prefer surgical methods in the treatment of PSD regardless of gender (14). The optimal surgical procedures for PSD should result in minimal length of hospital stay, satisfactory VAS scores, low disease recurrence, low complication rates, as much aesthetic satisfaction as possible, and allow patients to return to work $(15,16)$.

Complications and recurrence are the most important parameters for evaluating the effectiveness of a surgical method for PSD. From this point of view, it is seen that flap methods provide advantages against PMC with criteria such as low infection and recurrence rates (17-19). However, PMC method provides important 
advantages against other flap methods such as short hospital stay and a very positive aesthetic result. The most frequently reported disadvantage of flap methods is the high perception of tension with cosmetic and aesthetic concerns, and this can be very important for a woman (20). One of the most important result of our study is that these cosmetic concerns result more in flap methods.

In addition, when we analyse the literature especially in terms of complications, we especially determine seroma, and wound dehiscence $(21,22)$. In the current study, there were five occurrences of minimal seroma. One (3.44\%) of them was in PMC group and four (13.79\%) of them were in flap groups. This complication seems to be occurred due to the nature of the flap technique and because of larger tissue excision leading to bigger dead space comparing to PMC. These cases resolved by reccurant wound dressings and drainage. The other complications were separated equally and in two techniques and resolved by local antibiotherapy.

One of the limitations of this study is that being a retrospective study, and the other is the small sample size although it includes only female patients, which is first work with such feature in literature. Because of this, the statistics performed are not very significant. Another limitation is that, it was difficult to standardize because more than one surgeon operated the cases. Therefore, prospective randomized and multiple-case studies are needed to obtain more objective results.

\section{CONCLUSION}

The results of this study showed that female patients who underwent PMC had better satisfaction with the appearance of their scar. This supports the use of minimally invasive techniques and primary closure techniques in the treatment of female patients, whose PSD volumes are smaller than men, and the current behavior model of surgeons in PSD treatment. Although it is the first study in the literature which have only female PSD cases, further prospective studies in large patient populations are needed to determine the most effective treatment method of female PSD patients.

\section{ETHICAL DECLARATIONS}

Ethics Committee Approval: The study was carried out with the permission of Ethics Committee of Ankara Mevki Military Hospital (Permission granted: 08.05.2013, Decision no: 19).

Informed Consent: Because the study was designed retrospectively, no written informed consent form was obtained from patients.
Referee Evaluation Process: Externally peer-reviewed.

Conflict of Interest Statement: The authors have no conflicts of interest to declare.

Financial Disclosure: The authors declared that this study has received no financial support.

Author Contributions: All of the authors declare that they have all participated in the design, execution, and analysis of the paper, and that they have approved the final version.

\section{REFERENCES}

1. Sondenaa K, Andersen E, Nesvik I, Soreide JA. Patient characteristics and symptoms in chronic pilonidal sinus disease. Int J Colorectal Dis 1995; 10: 39-42.

2. Akinci OF, Kurt M, Terzi A, et al: Natal cleft deeper in patients with pilonidal sinus: Implications for choice of surgical procedure. Dis Colon Rectum 2009; 52: 1000-2.

3. Shareef SH, Hawrami TA, Salih AM, et al. Intermammary pilonidal sinus: the first case series. Int J Surg Case Rep 2017; 41: 265-8.

4. Spivak H, Brooks VL, Nussbaum M, Friedman I. Treatment of chronic pilonidal disease. Dis Colon Rectum 1996; 39: 1136-9.

5. Sinan H, Demirbas S, Ozer MT, Akyol M. Single-incision laparoscopic cholecystectomy versus laparoscopic cholecystectomy: A prospective randomized study. Surg Laparosc Endosc Percutan Tech 2012; 22: 12-6.

6. Sucullu I, Filiz AI, Canda AE, Yucel E, Kurt Y, Yildiz M. Body image and cosmesis after laparoscopic or open appendectomy. Surg Laparosc Endosc Percutan Tech 2009; 19: 401-4.

7. Maurice BA, Greenwood RK: A conservative treatment of pilonidal sinus. Br J Surg 1964; 51: 510-2.

8. Luedi MM, Kauf P, Evers T, et al. Impact of spinal versus general anesthesia on postoperative pain and long term recurrence after surgery for pilonidal disease. J Clin Anesth 2016; 33: 236-42.

9. Kazim D, Mustafa G, Ali H. Prevalence of sacrococcygeal pilonidal disease in Turkey. Asian J Surg 2017; 40: 434-7.

10. Sondenaa K, Andersen E, Nesvik I, Soreide JA. Patient characteristics and symptoms in chronic pilonidal sinus disease. Int J Colorectal Dis 1995; 10: 39e42.

11. Rahsan O, Mirzaman H, Ayten CB, Senol E, et al. Which treatment modality for pediatric pilonidal sinus: Primary repair or secondary healing? Asian J Surg 2018; 41: 506-10.

12. Kooistra HP. Pilonidal sinuses. Review of the literature and report of three hundred and fifty cases. Am J Surg. 1942; 55: $3 \mathrm{e} 17$.

13. Kanat BH, Girgin M. Etiologic factor in the increased frequency of pilonidal disease: computer DEU Med J 2013; 27: 59e61.

14. Çolak T, Sücüllü İ, Sinan H, Sengül N, Terzi C. Results of surgeon attitude questionnaire on pilonidal sinus. Kolon Rektum Hast Derg 2011; 21: 165-72.

15. Koorista HP. Pilonidal sinuses. Review of literature and report of three hundred and fifty cases. Am J Surg 1942; 55: 3-17.

16. Akıncı OF, Coskun A, Uzunköy A. Simple and effective surgical treatment of pilonidal sinus: asymmetric excision and primary closure using suction drain and subcuticular skin closure. Dis Colon Rectum 2000; 43: 701-7. 
17. Kapan M, Kapan S, Pekmezci S, Durgun V. Sacrococcygeal pilonidal sinus disease with Limberg flap repair. Tech Coloproctol 2002; 6: 27-32.

18. Bozkurt MK, Tezel E. Management of pilonidal sinus with the Limberg flap. Dis Colon Rectum 1998; 41: 775-7.

19. Mentes O, Bagci M, Bilgin T, Coskun I, Ozgul O, Ozdemir M. Management of pilonidal sinus disease with oblique excision and primary closure: results of 493 patients. Dis Colon Rectum 2006; 49: 104-8.

20. Eryilmaz R, Sahin M, Alimoglu O, Dasiran F. Surgical treatment of sacrococcygeal pilonidal sinus with the Limberg transposition flap. Surgery 2003; 134: 745-9.

21. Mentes O, Bagci M, Bilgin T, Coskun I, Ozgul O, Ozdemir M Management of pilonidal sinus disease with oblique excision and primary closure: results of 493 patients. Dis Colon Rectum 2006; 49: 104-18.

22. Mansoory A, Dickson D. Z-plasty for treatment of disease of the pilonidal sinus. Surg Gynecol Obstet 1982; 155: 409-11. 